\title{
Open-Label Adhesion Performance Studies of a New Lidocaine Topical System I.8\% versus Lidocaine Patches $5 \%$ and Lidocaine Medicated Plaster $5 \%$ in Healthy Subjects
}

This article was published in the following Dove Press journal:

Journal of Pain Research

\author{
Jeffrey Gudin ${ }^{1,2}$ \\ Lynn R Webster ${ }^{3}$ \\ Emileigh Greuber (D) $^{4}$ \\ Kip Vought ${ }^{4}$ \\ Kalpana Patel ${ }^{4}$ \\ Louis Kuritzky ${ }^{5}$
}

'Department of Anesthesiology, Rutgers New Jersey Medical School, Newark, NJ, USA; ${ }^{2}$ Department of Anesthesiology and Pain Management, Englewood Hospital and Medical Center, Englewood, NJ, USA; ${ }^{3}$ Scientific Affairs Neurosciences, PRA Health Sciences, Salt Lake City, UT, USA; ${ }^{4}$ Global Research and Development and Medical Affairs, Scilex Pharmaceuticals Inc, Palo Alto, CA, USA; ${ }^{5}$ Community Health and Family Medicine, University of Florida Community Health \& Family Medicine, Gainesville, FL, USA
Correspondence: Lynn R Webster Vice President Scientific Affairs, PRA Health Sciences, Salt Lake City, UT, 84103, USA

$\mathrm{Tel}+$ I 8019044593

Email WebsterLynn@Prahs.com
Purpose: The primary objective was to evaluate adhesion performance of the lidocaine topical system $1.8 \%$ for 12 hours in healthy human subjects in three studies: as a single product (Study 1) and versus other lidocaine topical products (lidocaine patch 5\% and lidocaine medicated plaster 5\% [Study 2] and generic lidocaine patch 5\% [Study 3]). Safety of the lidocaine topical system $1.8 \%$, with a skin irritation focus, was a secondary objective.

Patients and Methods: All three studies were open-label, randomized, Phase 1 adhesion performance studies in healthy adult volunteers $(\mathrm{N}=125)$. Lidocaine topical products were applied for 12 hours per test, per study arm. Adhesion of all test products was scored at 0,3 , 6,9 , and 12 hours post-application. Skin irritation was scored after product removal or when a product detached.

Results: Overall, the majority ( $\geq 75 \%$ ) of subjects treated with the lidocaine topical system $1.8 \%$ demonstrated $\geq 90 \%$ adhesion (FDA adhesion score 0 ) throughout the 12 -hour administration period versus $13.6 \%$ of subjects treated with lidocaine patch $5 \%, 15.9 \%$ of subjects treated with lidocaine medicated plaster $5 \%$, and $0 \%$ of subjects treated with the generic lidocaine patch $5 \%$. There were no complete detachments with the lidocaine topical system $1.8 \%$, whereas $4.5 \%$ of lidocaine patch $5 \%$ and lidocaine medicated plaster $5 \%$ detached, and $29 \%$ of generic lidocaine patch $5 \%$ detached. Minimal skin irritation was observed with each lidocaine topical product.

Conclusion: Across three studies, lidocaine topical system 1.8\% demonstrated superior adhesion performance versus the three other products tested. Skin irritation was minimal across products and studies.

Clinicaltrials.gov: NCT04312750, NCT04320173, NCT04319926.

Keywords: lidocaine topical system, lidocaine patch, lidocaine medicated plaster, adhesion, postherpetic neuralgia

\section{Introduction}

An estimated 1 million individuals in the United States are diagnosed with herpes zoster (shingles) each year following reactivation of a childhood infection with varicella-zoster virus. ${ }^{1,2}$ According to the US Centers for Disease Control and Prevention and health authorities in other countries, the incidence of herpes zoster has been gradually increasing in adults for several years, for reasons that are not well understood, although rates among older adults have plateaued since 2008. ${ }^{1}$ Postherpetic neuralgia 
(PHN) is the most common long-term complication of herpes zoster and is generally diagnosed in individuals who have recovered from the herpes zoster skin rash but continue to experience pain for at least 3 months. ${ }^{3}$ Depending on their age and the PHN definition used, $20 \%$ to $50 \%$ of individuals with herpes zoster will experience PHN. ${ }^{3,4}$ Advanced age and immunocompromised states are significant risk factors for developing both herpes zoster and PHN, as approximately half of all PHN cases occur in individuals aged $>60$ years. $^{3,4}$

PHN is characterized by neuropathic pain isolated to the dermatome(s) affected by the virus and can be debilitating. It is a chronic condition that can persist for years in some patients and is often challenging to treat. ${ }^{2-4}$ It is a major driver in herpes zoster-related disease burden and cost. ${ }^{5}$ Currently, there is no available cure for PHN; thus, medical management focuses on palliative treatments and strategies to shorten the duration or lessen the symptom burden. ${ }^{3}$ Due to the persistent and often refractory nature of the neuropathic pain associated with PHN, combination therapy with systemic and topical analgesics is often utilized in an attempt to optimize patient outcomes. ${ }^{2}$ Systemic therapies may include antidepressants, anticonvulsants, and opioids; opioids have remained the most frequently used first-line therapy, despite lack of support by the current body of evidence and potential for abuse or diversion.,

Topical patches and other delivery systems have been developed to deliver drugs, such as lidocaine, locally for the treatment of PHN..$^{2-47}$ Topical delivery may have clinical advantages, including specifically targeting the site of pain with the delivery of drugs to the affected area, thereby posing a low risk of systemic toxicity or drug-drug interactions and potentially avoiding some of the adverse events (AEs) associated with commonly used systemic medications. ${ }^{7-9}$ This is particularly important in older patients, who may have impaired hepatic and/or renal function, and for those in whom AEs can be a limiting factor in treatment compliance. ${ }^{3}$

Lidocaine is an amide-type local anesthetic agent that stabilizes neuronal membranes by inhibiting the ionic fluxes required for initiation and conduction of nerve impulses (ie, pain signals). ${ }^{10}$ Because topically applied lidocaine is reported to penetrate only about 8 to $10 \mathrm{~mm}$ into the skin, it is well suited for targeted use in peripherally localized pain. ${ }^{11,12}$ Penetration of lidocaine after application of the lidocaine patch 5\% was sufficient to produce an analgesic effect, but less than the amount necessary to produce a complete sensory block. $^{13}$ In 1999, the US Food and Drug Administration
(FDA) granted approval for Lidoderm ${ }^{\circledR}$ (lidocaine patch $5 \%$; Endo Pharmaceuticals Inc., Malvern, PA) as the first medication specifically indicated for the treatment of PHN. ${ }^{8,13,14}$ The drug product was approved in Europe in 2007, designated as lidocaine "medicated plaster". ${ }^{15}$ Generic lidocaine patch 5\% products were introduced to the market by Actavis Laboratories UT Inc. (Salt Lake City, UT) in August 2012 and Mylan Pharmaceuticals Inc. (Morgantown, WV) in August 2015. ${ }^{16,17}$

FDA recently moved to use of a standardized nomenclature for topical or transdermal dosage forms, referred to as "systems" instead of "patches." In February 2018, FDA approved ZTlido ${ }^{\circledR}$ (lidocaine topical system 1.8\%; Scilex Pharmaceuticals Inc., San Diego, CA), which is indicated for relief of pain associated with PHN. ${ }^{10}$ The lidocaine topical system $1.8 \%$, lidocaine medicated plaster $5 \%$, and lidocaine patch $5 \%$ products are all topical dosage forms (ie, they all deliver lidocaine topically to the site of pain). The lidocaine topical system $1.8 \%$ is a thin and flexible anhydrous lidocaine delivery system that was developed to provide transcutaneous lidocaine exposure equivalent to the prescription lidocaine $5 \%$ products (lidocaine patch $5 \%$ and lidocaine medicated plaster $5 \%$ in the United States and European Union, respectively), with a significantly reduced drug load per system (36 mg vs $700 \mathrm{mg}) .{ }^{10,13}$ Approval of the lidocaine topical system $1.8 \%$ was based, in part, on its demonstrated bioequivalence to the lidocaine patch $5 \%$, allowing for comparable pain relief with greater bioavailability of lidocaine. ${ }^{18,19}$ Thus, with the same surface area, $140 \mathrm{~cm}^{2}$, one dose of the lidocaine topical system $1.8 \%$ provides lidocaine exposure equivalent to one lidocaine patch $5 \%{ }^{10,19}$

Although it has been established that topical lidocaine provides effective analgesia for individuals with PHN, it is generally understood that the adhesive performance of a topical or transdermal delivery system is an essential factor in bioavailability because suboptimal adhesion may affect drug exposure and achievement of a therapeutic dose. ${ }^{20,21}$ FDA has received numerous reports of adhesion failureincluding edge curling, partial lifting, or complete detachment of patches - that have resulted in improper dosing as well as repeat application, leading to increased cost. Approximately $69 \%$ of AEs reported to the FDA Adverse Event Reporting System for topical lidocaine 5\% patches from 2013 to 2018 were related to product adhesion issues (1347 adhesion issues out of 1936 reported total AE cases). ${ }^{22}$

Three studies were conducted to characterize the adhesion of the lidocaine topical system $1.8 \%$. The first study was 
designed to assess the adhesion performance of the topical system in healthy human subjects over 12 hours, a maximum application period described in the product labeling. ${ }^{10}$ The subsequent two studies then compared the adhesion performance of the lidocaine topical system $1.8 \%$ to that of three other prescription products in common use: lidocaine patch $5 \%$, and lidocaine medicated plaster 5\% in Study 2, as well as a newer US generic lidocaine patch 5\% from Mylan Pharmaceuticals Inc. in Study 3 (Table 1). Safety of the lidocaine topical system $1.8 \%$ was also monitored throughout the studies.

\section{Methods}

\section{Study Design}

All three studies were Phase 1 adhesion performance studies. Study 1 was an open-label, single-treatment, single-period, single-application study; Study 2 was an open-label, randomized, single-treatment, 3-period, single-application study; and Study 3 was an open-label, randomized, 2-treatment, 2-period, single-dose study. The studies were conducted at single clinical sites in the United States: Studies 1 and 3 at AXIS Clinicals, Dilworth, MN, and Study 2 at TKL Research Inc., Fair Lawn, NJ. Study 1 was approved by the Salus Institutional Review Board, and Studies 2 and 3 were approved by the IntegReview Board (both central IRBs in Austin, TX). All three studies were conducted in accordance with the ethical principles originating from the Declaration of Helsinki and amendments, ICH Guideline for Good Clinical Practice, FDA Guidance for Industry: Assessing Adhesion With Transdermal and Topical Delivery Systems for ANDAs, and local regulatory requirements. ${ }^{23,24}$ Study 2 was also performed in accordance with Appendix II (In Vivo Skin Adhesion) of the European Medicines Agency Guideline on the Pharmacokinetic and Clinical Evaluation of Modified Release Dosage Forms. ${ }^{25}$ These studies were registered at Clinicaltrials.gov with the clinical trial registration numbers: NCT04312750, NCT04320173, and NCT04319926.

\section{Subjects}

The studies were performed in healthy adult human volunteers with similar inclusion and exclusion criteria. Eligible subjects were men or women $\geq 18$ years of age, with a body mass index between 18 and $36 \mathrm{~kg} / \mathrm{m}^{2}$ (Study 1) or 18 and $30 \mathrm{~kg} / \mathrm{m}^{2}$ (Studies 2 and 3). Subjects were nonsmokers and generally healthy, as documented by medical history, physical examination (including evaluation of cardiovascular, gastrointestinal, respiratory, musculoskeletal, and central nervous systems), and vital signs assessments, with no evidence of underlying disease during check-in and screening performed within 28 days of check-in. Female subjects could not be pregnant or lactating, and those of childbearing age were instructed to practice medically acceptable contraception throughout the study. All subjects provided prior written informed consent and were able to comply with study procedures.

Exclusion criteria included evidence of allergy or known hypersensitivity to lidocaine, local anesthetics of the amide type, or any of the components of the lidocaine topical system formulations; any major illness in the last 3 months or any significant ongoing chronic medical illness; history of addiction, abuse, and misuse of any drug; alcohol abuse within the prior 12 months; ingestion of prescription medication or any hormonal medication (except hormonal contraceptives) at any time in 14 days prior to study product application; and use of over-the-counter medications within the prior 5 days.

Table I Characteristics of Studied Lidocaine Topical Products

\begin{tabular}{|c|c|c|c|c|}
\hline & $\begin{array}{l}\text { Lidocaine Topical System } \\
\text { I.8\% }\end{array}$ & Lidocaine Patch 5\% & $\begin{array}{l}\text { Lidocaine Medicated } \\
\text { Plaster } 5 \%\end{array}$ & Mylan Lidocaine Patch 5\% \\
\hline Formulation & Nonaqueous polymer & Hydrogel & Hydrogel & Nonaqueous polymer \\
\hline $\begin{array}{l}\text { Lidocaine } \\
\text { content }\end{array}$ & $\begin{array}{l}36 \mathrm{mg} \text { (18 } \mathrm{mg} \text { per gram of } \\
\text { adhesive: } 1.8 \% \text { ) }\end{array}$ & $\begin{array}{l}700 \mathrm{mg}(50 \mathrm{mg} \text { per gram of } \\
\text { adhesive: } 5 \% \text { ) }\end{array}$ & $\begin{array}{l}700 \mathrm{mg} \text { ( } 50 \mathrm{mg} \text { per gram of } \\
\text { adhesive: } 5 \%)\end{array}$ & $\begin{array}{l}140 \mathrm{mg} \text { ( } 50 \mathrm{mg} \text { per gram of } \\
\text { adhesive: } 5 \%)\end{array}$ \\
\hline Bioavailability & $\sim 45 \%$ & $\sim 3 \pm 2 \%$ & $\sim 3 \pm 2 \%$ & $\sim 11 \pm 4 \%$ \\
\hline Residual drug & $\sim 20 \mathrm{mg}$ & $\geq 665 \mathrm{mg}$ & $\geq 665 \mathrm{mg}$ & $\sim 115 \mathrm{mg}$ \\
\hline Release liner & Perforated & Not perforated & Not perforated & Not perforated \\
\hline Size & $10 \mathrm{~cm} \times 14 \mathrm{~cm}$ & $10 \mathrm{~cm} \times 14 \mathrm{~cm}$ & $10 \mathrm{~cm} \times 14 \mathrm{~cm}$ & $10 \mathrm{~cm} \times 14 \mathrm{~cm}$ \\
\hline Thickness & $0.08 \mathrm{~cm}$ & $0.16 \mathrm{~cm}$ & $0.16 \mathrm{~cm}$ & $0.02 \mathrm{~cm}$ \\
\hline Weight & $2 \mathrm{~g}$ adhesive & $14 \mathrm{~g}$ adhesive & $14 \mathrm{~g}$ adhesive & $2.8 \mathrm{~g}$ adhesive \\
\hline
\end{tabular}

Notes: ZTLIDO ${ }^{\circledR}$ (lidocaine topical system) 1.8\% Prescribing Information. II/20I8. LIDODERM ${ }^{\circledR}$ (lidocaine patch 5\%) Prescribing Information. 0I/20I5. VERSATIS ${ }^{\circledR}$ (lidocaine medicated plaster 5\%) Prescribing information. 07/20।8. Mylan Lidocaine Patch 5\% Prescribing information. 0 I/20I5. 
Of relevance to dermatologic adhesion studies, subjects could not have the following: history of significant dermatologic cancers (eg, melanoma, squamous cell carcinoma), except basal cell carcinomas that were superficial and did not involve the investigative site; presence of any current dermatologic condition (eg, psoriasis, eczema, atopic dermatitis) or any skin condition such as scratches, cuts, scars, abrasions, excessive hair, tattoos, moles, recently shaved skin, uneven skin texture, irritated skin (redness, rash, blisters, etc.), or excessively oily skin at the application areas that may have affected the application or adhesive properties of the study products; and medical history of hyperhidrosis, or otherwise history of excessive sweating under non-exercising conditions.

\section{Treatments}

Prior to topical product application, the hair on the application site was clipped if necessary (not shaved) by clinical staff. The area was checked to ensure the absence of any skin conditions (eg, broken skin, moles, uneven skin texture) and was cleaned only with water (no soaps or cleansing agents) and allowed to air-dry. Each product was applied away from any significant folds or creases and at least 1 inch away from the spine. The application involved pressing the product firmly into place, holding it with the palm of the hand for up to 15 seconds, and smoothing it to prevent trapping of air bubbles. No overlays, adhesive tapes, bandages, or similar products were applied during the application period.

In Study 1, a single lidocaine topical system $1.8 \%$ was applied to a fixed area on either the left or right side (per randomization schedule) of the lower/middle back. In Study 2, one of the three products, the lidocaine topical system $1.8 \%$, lidocaine patch $5 \%$, or lidocaine medicated plaster 5\%, was applied over a predetermined fixed area on either the left or right side of the upper back (per randomization schedule). Each subject received all three products (one in each study period), with the order of products determined by randomization to one of six treatment sequences. The lidocaine topical system $1.8 \%$ was randomized to be applied on one side, and the lidocaine patch $5 \%$ or lidocaine medicated plaster $5 \%$ was applied contralaterally at the same anatomical location during their respective subsequent periods. In Study 3, one lidocaine topical system $1.8 \%$ or one generic lidocaine patch $5 \%$ was applied over a predetermined fixed area on the upper back (per randomization schedule). Each subject received both products (one in each application period), with the order of products determined by randomization.
Throughout all studies, products were worn for 12 hours ( \pm 15 minutes) in each application period, which occurred during daylight hours (morning to evening). Products were removed and discarded per protocol guidelines, and the application site was gently wiped with dry gauze. Washout intervals occurred between consecutive application periods when more than one product was evaluated: a 7-day washout was used between periods I and II and periods II and III in Study 2, and a 12-hour washout was used between periods I and II in Study 3.

\section{Subject Compliance}

The use of soap or topical products (eg, lotion, oil, makeup, powder) was not permitted on the study product application site for 48 hours prior to product application and throughout the entire duration of the study. Subjects were instructed not to wash off or wet the application area until after the 12-hour post-removal irritation assessment was performed. They were instructed to avoid rubbing, pulling, scratching, or touching the product or performing any other activity that might cause the product to be displaced. Specifically, they were to avoid putting pressure between the product and other objects (eg, walls, chairs, beds) for prolonged periods. They could not participate in any strenuous activity but could ambulate and perform activities freely if these required no physical exertion or did not hamper product adhesion. They were not allowed to press down or re-adhere any product that was lifting or detaching. Subjects were also required to avoid exposing the product to external sources of direct heat (eg, hair dryers, heating pads, heat lamps, saunas).

\section{Prior and Concomitant Therapy}

Subjects could not use prescription medications, except for hormonal contraception, within 14 days prior to the first product application and throughout the study. Over-thecounter medications were not allowed within the prior 5 days, but occasional use of acetaminophen, supplements, and vitamins was permitted. In addition, subjects refrained from using antiarrhythmic drugs, such as tocainide and mexiletine, and local anesthetics within 14 days prior to product application and throughout the study. Finally, subjects were prohibited from using nicotine-containing products (eg, e-cigarettes, patches, gum, chewing tobacco) within 30 days prior to product application and from consuming alcohol and poppy seed-containing foods within 48 hours prior to product application. 


\section{Study Objectives}

The primary objective of these studies was to evaluate the adhesion performance of the lidocaine topical system $1.8 \%$ for 12 hours in healthy human subjects: as a single product (Study 1) and in comparison with other available topical products (lidocaine patch $5 \%$ and lidocaine medicated plaster 5\% [Study 2] and generic lidocaine patch 5\% [Study 3]) An additional secondary objective throughout all studies was to monitor AEs and ensure the safety of the lidocaine topical system $1.8 \%$, with a focus on skin irritation.

\section{Study Assessments}

\section{Adhesion}

In all studies, product adhesion was assessed immediately after application ( 0 hours) and at 3, 6, 9, and 12 hours ( \pm 15 minutes; before product removal) after application. Assessments in Study 1 were performed by a trained scorer using the FDA-recommended 5-point adhesion scale. ${ }^{24}$ The FDA scale ranges from 0 to 4 , where 0 represents $\geq 90 \%$ of the product adhered (essentially no part of the product lifting off the skin), 1 represents $75 \%$ to $<90 \%$ adhered (only some edges of the product lifting off the skin), 2 represents $50 \%$ to $<75 \%$ adhered (less than half the product lifting off the skin), 3 represents $>0 \%$ to $<50 \%$ adhered (more than half the product lifting off the skin but not detached), and 4 represents $0 \%$ adhered (complete product detachment). The mean cumulative adhesion score was calculated by summing the scores at 3, 6, 9, and 12 hours and dividing the total by the total number of observations per subject.

In Studies 2 and 3, the degree of adhesion was assessed by a trained scorer using a transparent grid with evenly spaced dots. The grid was demarcated to the exact size of each lidocaine product. The dot matrix grid was gently laid over the product on the skin, and areas of adhesion were outlined; dots excluded from adhering areas were counted to identify the exact surface area of lift-off and allowing the determination of the total amount of product adhesion as a percentage (ie, percent adhesion). In Study 2, the percent adhesion for each individual product was also transposed to the FDA 5-point scale as the primary dataset for analysis. However, an ad-hoc analysis of the percent adhesion data for each product was also performed as it is more discrete in detecting differences in adhesion performance. In Study 3, the degree of adhesion for both lidocaine products was assessed using the dot matrix method described above and reported as total percent adhesion with no transposition to other scales.
If a subject reported that a product was becoming detached, adhesion assessments were performed within 10 minutes. For products that had completely detached prior to the end of a 12-hour application period in Studies 2 and 3, a score of $0 \%$ was carried through in the adhesion analysis for all remaining observations in that application period.

\section{Safety}

In Study 1, skin irritation at the application site of the lidocaine topical system $1.8 \%$ was evaluated at 30 minutes ( +10 minutes) and 2 hours ( \pm 15 minutes) after product removal using an 8-point scale of dermal response (where 0 represents no evidence of irritation; scores then range from 1 [representing mild, barely perceptible erythema] to 7 [representing a strong reaction, extending beyond the application site]) and a scale of other effects, including glazed appearance, peeling and cracking, dried or serous exudates covering at least a portion of the application site, and small petechial erosions and/or scabs). ${ }^{26}$ In Study 2, skin irritation at the application site was also evaluated at 30 minutes ( +15 minutes), 2 hours ( \pm 15 minutes), and 12 hours ( \pm 30 minutes) after product removal to confirm whether any irritation observed immediately after removal was associated with mechanical removal of the patch. If the product was detached or otherwise removed prior to the conclusion of the 12hour application period, an irritation assessment was made at the time of detachment and at all subsequent time points in what would have been the full 12-hour application period had detachment not occurred. AEs were monitored and recorded at specified time points throughout the study. In Study 3, dermal AEs were reported, but no formal skin irritation assessments were performed.

\section{Statistical Analyses}

In Study 1, descriptive statistics (mean, standard deviation, median, minimum, and maximum) for cumulative adhesion score and mean cumulative adhesion score were generated. Statistical analysis was performed using $\operatorname{SAS}^{\circledR}$ Version 9.4 (SAS Institute Inc., Cary, NC). In Study 2, mean adhesion scores were analyzed using analysis of variance (ANOVA) with effects of sequence, subjects within sequence, period, and treatment. Sequence was tested against subjects within sequence for significance $(P<0.05)$; period and treatment were tested against the residual error $(P<0.05)$. A 2 -sided $90 \%$ confidence interval (CI; equivalent to two 1 -sided $95 \%$ tests) was constructed on the difference (test minus reference) between treatment means. Proportions of subjects for secondary endpoints 
were compared between treatment groups using CochranMantel-Haenszel statistics blocking on subject. In Study 3, statistical significance for a treatment effect was determined by ANOVA. A sample size of 24 for Study 3 was calculated for this 2-way crossover design based on the analysis of Study 2. Because the generic lidocaine patch $5 \%$ is required to demonstrate comparable (statistically noninferior) adhesion to the reference product lidocaine patch $5 \%$ used in Study 2 to support its marketing approval, it was assumed that Study 3 would be adequately powered at $80 \%$ with 24 subjects, based on the effect size and coefficient of variation observed for lidocaine patch $5 \%$ in Study 2. Statistical analysis was performed using SAS $^{\circledR}$ Version 9.3 (SAS Institute Inc., Cary, NC).

\section{Results}

\section{Subject Disposition and Baseline Characteristics}

A total of 125 subjects were randomized in these studies, and all received treatment. In Study 1, 54 were enrolled and completed the study. In Study 2, 47 subjects were enrolled and $44(93.6 \%)$ completed the study, while 3 $(6.4 \%)$ discontinued when they were unable to reach the study site due to severe weather. In Study 3, 24 subjects were enrolled and completed the study. Throughout, no subjects discontinued due to AEs (eg, unacceptable skin irritation) or were excluded due to a protocol deviation, and 122 subjects who completed the studies were included in the per-protocol adhesion analyses (54, 44, and 24 subjects in Studies 1, 2, and 3, respectively).

Overall, subjects ranged from 18 to 64 years of age. The majority of subjects were female in Studies 1 (70.4\%) and $3(62.5 \%)$, while male subjects predominated in Study $2(74.5 \%)$. Across the three studies, the majority of subjects were white $(85.2 \%, 46.8 \%$, and $62.5 \%$ in Studies 1 , 2 , and 3, respectively) (Table 2). All subjects were healthy, with vital signs within the normal range.

\section{Adhesion Analysis}

\section{Study I}

Among the 54 subjects included in the analysis, 27 received the lidocaine topical system $1.8 \%$ on the left side of the lower/ middle back and 27 received the product on the right side. Adhesion evaluations performed every 3 hours during the 12 hours of administration used the FDA rating scale. Overall, mean cumulative adhesion scores across all time points ranged

Table 2 Subject Demographics and Baseline Characteristics

\begin{tabular}{|c|c|c|c|}
\hline Characteristics & $\begin{array}{l}\text { Study I } \\
(n=54)\end{array}$ & $\begin{array}{l}\text { Study } 2 \\
(n=47)\end{array}$ & $\begin{array}{l}\text { Study } 3 \\
(n=24)\end{array}$ \\
\hline \multicolumn{4}{|l|}{ Age, years } \\
\hline Mean (SD) & $30.1(12.5)$ & $43.1(10.8)$ & 34.79 (13.39) \\
\hline Median & 25.0 & 47.0 & 32.00 \\
\hline Range & $18-58$ & $19-59$ & $19-64$ \\
\hline \multicolumn{4}{|l|}{ Sex, n (\%) } \\
\hline Male & $16(29.6 \%)$ & 35 (74.5\%) & 9 (37.5\%) \\
\hline Female & 38 (70.4\%) & $12(25.5 \%)$ & 15 (62.5\%) \\
\hline \multicolumn{4}{|l|}{ Race, n (\%) } \\
\hline White & 46 (85.2\%) & $22(46.8 \%)$ & 15 (62.5\%) \\
\hline Black & 5 (9.3\%) & 21 (44.7\%) & $4(16.7 \%)$ \\
\hline Asian & $2(3.7 \%)$ & $3(6.4 \%)$ & 3 (I2.5\%) \\
\hline Other & I (1.9\%) & I (2.1\%) & $0(0 \%)$ \\
\hline
\end{tabular}

Abbreviation: SD, standard deviation.

Table 3 Mean Adhesion Scores Using FDA Scales for Lidocaine Topical System I.8\%, Lidocaine Patch 5\%, and Lidocaine Medicated Plaster (Studies I, 2, and 3)

\begin{tabular}{|c|c|c|c|c|c|c|}
\hline \multirow[b]{2}{*}{ Parameters } & \multirow{2}{*}{$\begin{array}{l}\text { Study I } \\
\text { Lidocaine } \\
\text { Topical System } \\
\text { I.8\% }(n=54)\end{array}$} & \multicolumn{3}{|l|}{ Study 2} & \multicolumn{2}{|l|}{ Study 3* } \\
\hline & & $\begin{array}{l}\text { Lidocaine } \\
\text { Topical System } \\
\text { I.8\% }(n=44)\end{array}$ & $\begin{array}{l}\text { Lidocaine } \\
\text { Patch 5\% } \\
(n=44)\end{array}$ & $\begin{array}{l}\text { Lidocaine } \\
\text { Medicated } \\
\text { Plaster 5\% } \\
(n=44)\end{array}$ & $\begin{array}{l}\text { Lidocaine } \\
\text { Topical System } \\
\text { I.8\% }(n=24)\end{array}$ & $\begin{array}{l}\text { Generic } \\
\text { Lidocaine } \\
\text { Patch 5\% } \\
(n=24)\end{array}$ \\
\hline $\begin{array}{l}\text { Mean cumulative } \\
\text { adhesion score (FDA } \\
\text { scale), mean } \pm \text { SD }\end{array}$ & $0.04 \pm 0.138$ & $0.20 \pm 0.38$ & $1.01 \pm 0.66$ & $0.89 \pm 0.66$ & $0.17 \pm 0.48$ & $2.63 \pm 0.58$ \\
\hline Minimum score* & 0 & 0 & 0 & 0 & 0 & 0 \\
\hline Maximum score* & I & 3 & 4 & 4 & 2 & 4 \\
\hline
\end{tabular}

Note: *Percent adhesion data were converted to corresponding FDA adhesion rating scale scores for Study 3. 
from 0 ( $>90 \%$ adhered) to 1 ( $\geq 75 \%$ to $<90 \%$ adhered), and the mean of these scores was $0.04 \pm 0.138$ (Table 3).

More than $90 \%$ of subjects had a score of 0 at every time point from 0 to 12 hours after application. At the end of the 12hour study period, 49 of 54 subjects $(90.7 \%)$ had a score of 0 , while the 5 remaining subjects had a low score of 1 , indicating that only some edges of the lidocaine topical delivery system $1.8 \%$ lifted off the skin (Figure 1A). No subject had a meaningful degree of detachment (score $\geq 3$ ). Cumulatively across all time points, 47 subjects $(87 \%)$ had an adhesion score of 0 for all evaluations performed every 3 hours during the 12 hours of administration, 7 subjects (13\%) had an adhesion

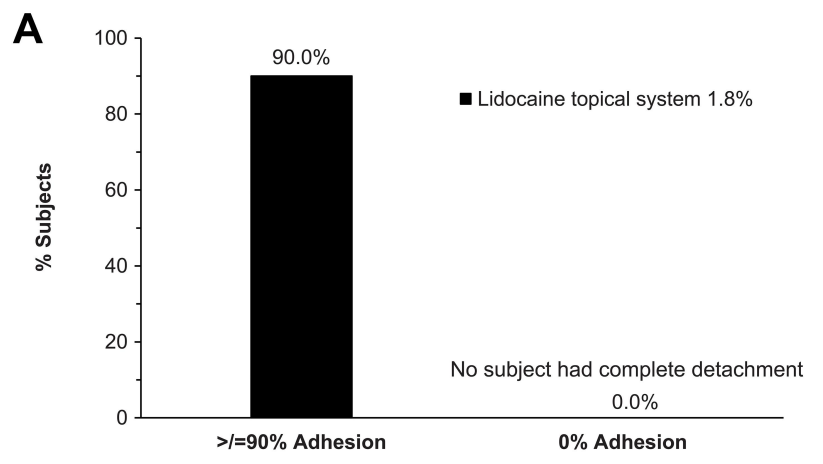

B
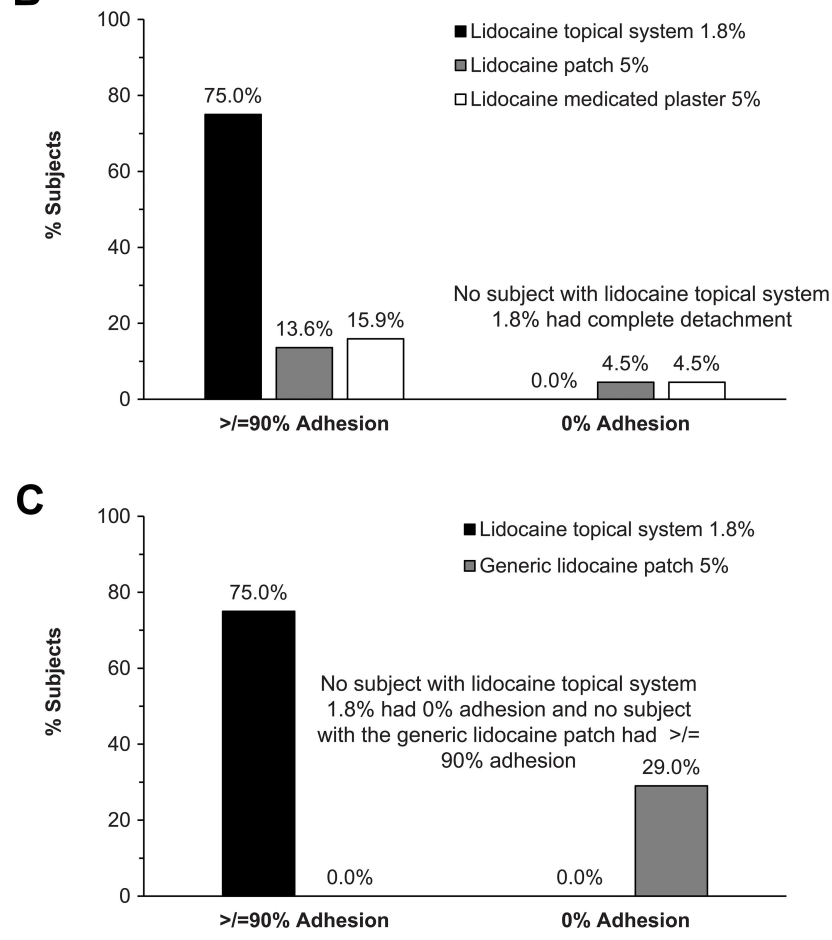

Figure I Adhesion assessments at the end of the dosing period ( 12 hours). The proportion of subjects with at least $90 \%$ adhesion (using the FDA adhesion rating scale) at the end of the 12-hour application period and the proportion of subjects who had experienced $0 \%$ adhesion (complete detachment) at any time point throughout the study in (A) Study I ( $n=54)$; (B) Study $2(n=44)$; and (C) Study $3(n=24)$. score of 1 ( $\geq 75 \%$ to $<90 \%$ adhered) for at least one evaluation, and no subject had a score of 2 or greater ( $<75 \%$ adhered).

\section{Study 2}

A total of 47 subjects were randomized to 1 of 6 treatment sequences specifying the order of application of the 3 products, and 44 subjects who completed the study were included in the analysis. Lidocaine topical system $1.8 \%$ showed a superior adhesion profile compared to both lidocaine patch $5 \%$ and lidocaine medicated plaster 5\% (Tables 3 and 4). The mean cumulative adhesion score using the FDA-rating scale was $0.20 \pm 0.38$ for lidocaine topical system $1.8 \%$, compared to $1.01 \pm 0.66$ and $0.89 \pm 0.66$ for the lidocaine patch $5 \%$ and lidocaine medicated plaster 5\%, respectively. At the 0-hour time point, the mean (90\% CI) adhesion score, determined using the FDA rating scale, was $0.003(-0.040,0.047)$ for the lidocaine topical system $1.8 \%, 0.049(0.006$, $0.093)$ for the lidocaine patch 5\%, and 0.047 (0.004, $0.091)$ for lidocaine medicated plaster 5\%. These scores were not significantly different $(P=0.2141$ and $P=0.2350$ for the lidocaine topical system $1.8 \%$ vs lidocaine patch $5 \%$ and lidocaine medicated plaster 5\%, respectively); however, all subsequent time points showed superior adhesion of the lidocaine topical system $1.8 \%$ versus both the lidocaine patch $5 \%$ and lidocaine medicated plaster 5\% $(P<0.0001$ for all comparisons $)$.

Analysis of the mean percent adhesion score determined from the raw data showed the superior adhesion of the lidocaine topical system $1.8 \%$ over both the lidocaine patch $5 \%$ and lidocaine medicated plaster $5 \%$ at baseline (ie, Time 0 ) and at each time point from 3 to 12 hours after application $(P<0.0001$ for all comparisons) (Figure 2A and Table 4). In contrast to analyses of FDA rating scale data, this analysis of percent adhesion data discretely revealed the superiority of the lidocaine topical system $1.8 \%$ at the 0 -hour time point (ie, immediate difference after product application).

At each time point during the 12-hour application period, a greater proportion of subjects had an FDA adhesion score of 0 ( $\geq 90 \%$ adhered) for the lidocaine topical system $1.8 \%$ compared with the lidocaine patch $5 \%$ or lidocaine medicated plaster 5\%. At 12 hours after application, a significantly higher number of subjects had an FDA adhesion score of 0 ( $\geq 90 \%$ adhered) for the lidocaine topical system 1.8\% (33 subjects, $75.0 \%$ ) than for the lidocaine patch $5 \%$ (6 subjects, $13.6 \%$ ) or lidocaine medicated plaster 5\% (7 subjects, $15.9 \%$ ) (Figure 1B). 
Table 4 Mean Percent Adhesion Over Time for Lidocaine Topical System I.8\% and Lidocaine Patch/Plaster 5\% Comparators (Studies 2 and 3)

\begin{tabular}{|c|c|c|c|c|c|}
\hline \multirow[b]{2}{*}{$\begin{array}{l}\text { Mean Percent } \\
\text { Adhesion Score }\end{array}$} & \multicolumn{3}{|l|}{ Study 2} & \multicolumn{2}{|l|}{ Study 3} \\
\hline & $\begin{array}{l}\text { Lidocaine Topical } \\
\text { System I.8\% }(n=44)\end{array}$ & $\begin{array}{l}\text { Lidocaine } \\
\text { Patch 5\% } \\
(n=44)\end{array}$ & $\begin{array}{l}\text { Lidocaine Medicated } \\
\text { Plaster 5\% }(n=44)\end{array}$ & $\begin{array}{l}\text { Lidocaine Topical } \\
\text { System I.8\% }(n=24)\end{array}$ & $\begin{array}{l}\text { Generic Lidocaine } \\
\text { Patch 5\% }(n=24)\end{array}$ \\
\hline 0 hours (mean \pm SD) & $100.0 \pm 0.0$ & $97.2 \pm 2.9$ & $97.2 \pm 3.9$ & $99.5 \pm 0.7$ & $77.6 \pm 17.3$ \\
\hline 3 hours (mean \pm SD) & $96.9 \pm 7.3$ & $84.7 \pm 13.1$ & $86.0 \pm 15.0$ & $97.0 \pm 4.8$ & $51.9 \pm 22.2$ \\
\hline 6 hours (mean \pm SD) & $95.0 \pm 8.6$ & $78.5 \pm 18.0$ & $80.9 \pm 16.8$ & $96.3 \pm 5.5$ & $40.8 \pm 22.9$ \\
\hline 9 hours (mean \pm SD) & $91.8 \pm 10.5$ & $70.9 \pm 22.2$ & $75.7 \pm 20.0$ & $94.7 \pm 7.1$ & $31.2 \pm 23.4$ \\
\hline 12 hours (mean $\pm S D$ ) & $88.7 \pm 13.8$ & $63.3 \pm 24.2$ & $68.4 \pm 24.4$ & $93.4 \pm 7.2$ & $26.8 \pm 23.0$ \\
\hline $\begin{array}{l}\text { Mean cumulative } \\
\text { adhesion \% (mean } \pm \\
\text { SD) }\end{array}$ & $93.1 \pm 9.8$ & $74.4 \pm 18.3$ & $77.8 \pm 17.4$ & $95.3 \pm 5.9$ & $37.7 \pm 21.3$ \\
\hline
\end{tabular}

Taken together, all analyses after the 0-hour time point showed superior adhesion of the lidocaine topical system $1.8 \%$ compared with the other products. Furthermore, no lidocaine topical system $1.8 \%$ completely detached during this study, whereas 2 each of both the lidocaine patch $5 \%$ and lidocaine medicated plaster 5\% completely detached (Figure 1B).

Photographs depicting adhesion of the lidocaine topical system $1.8 \%$, lidocaine patch $5 \%$, and lidocaine medicated plaster 5\% were taken immediately following product application at 0 hours and after 12 hours ( \pm 15 minutes) of wear. Representative images are shown in Figure 3A.

\section{Study 3}

All 24 subjects who were randomized to one of the two treatment sequences that specified the order of application of the two products were included in the analysis. Among subjects treated with the lidocaine topical system $1.8 \%$, the direct mean $(95 \% \mathrm{CI})$ percent adhesion score over time was $95.33 \%$ (89.40\% and $101.26 \%$ ) versus $37.67 \%$ (31.74\% and $43.60 \%$ ) for the generic lidocaine patch $5 \%$ (Table 4$)$. This difference was statistically significant $(P<0.0001)$. All subjects treated with the lidocaine topical system 1.8\% (24 of 24 [100\%]) demonstrated mean percent adhesion scores that were greater than those for subjects treated with the generic lidocaine patch $5 \%$.

Subjects treated with the lidocaine topical system $1.8 \%$ were able to maintain a mean percent adhesion score approaching $100 \%$ throughout the duration of the 12-hour assessment period. In contrast, the mean percent adhesion of the generic lidocaine patch $5 \%$ was approximately $80 \%$ immediately after application, and this decreased to below $40 \%$ over the 12 hours of the application period (Figure $2 \mathrm{~B}$ ). At each assessment time point, adhesion scores were significantly greater for the lidocaine topical system $1.8 \%$ than for the generic lidocaine patch $5 \%(P<0.0001$ at $0,3,6,9$, and 12 hours after application).

Only 6 of $24(25 \%)$ subjects treated with the lidocaine topical system $1.8 \%$ demonstrated an adhesion score $<90 \%$ at 12 hours. In contrast, 24 of $24(100 \%)$ subjects treated with the generic lidocaine patch $5 \%$ had scores below this level at 12 hours (ie, no subject had $\geq 90 \%$ adhesion).

In addition, $37.5 \%$ of subjects experienced substantial detachment (to $<10 \%$ adhesion) while using the generic lidocaine patch 5\%, including 7 (29.1\%) complete detachments (Figure 1C). Representative photographs comparing adhesion of the lidocaine topical system $1.8 \%$ and generic lidocaine patch 5\% following product application at 0 hours and after 12 hours ( \pm 15 minutes) of wear are shown in Figure $3 \mathrm{~B}$.

\section{Safety}

A comprehensive assessment of skin irritation was performed in Study 1 at 0.5 ( +10 minutes) and 2 hours $( \pm 15$ minutes) after removal of the lidocaine topical system $1.8 \%$. The mean irritation score at 0.5 hours was $0.52 \pm$ 0.64 , well below a score of 1 , which indicates mild, barely perceptible erythema. The score significantly decreased to $0.30 \pm 0.46$ by 2 hours $(P=0.0092)$. Four subjects $(7.4 \%)$ were observed to have an irritation score of 2 (definite erythema, minimal edema, or minimal papular response) at 
A

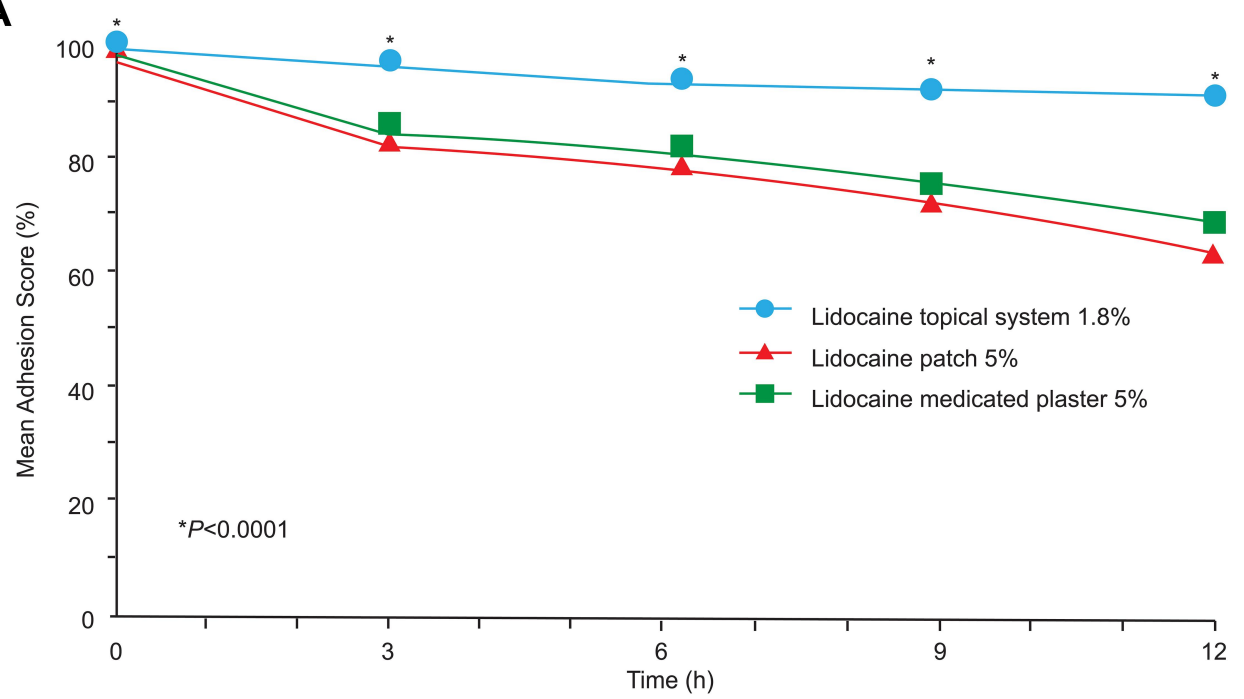

B

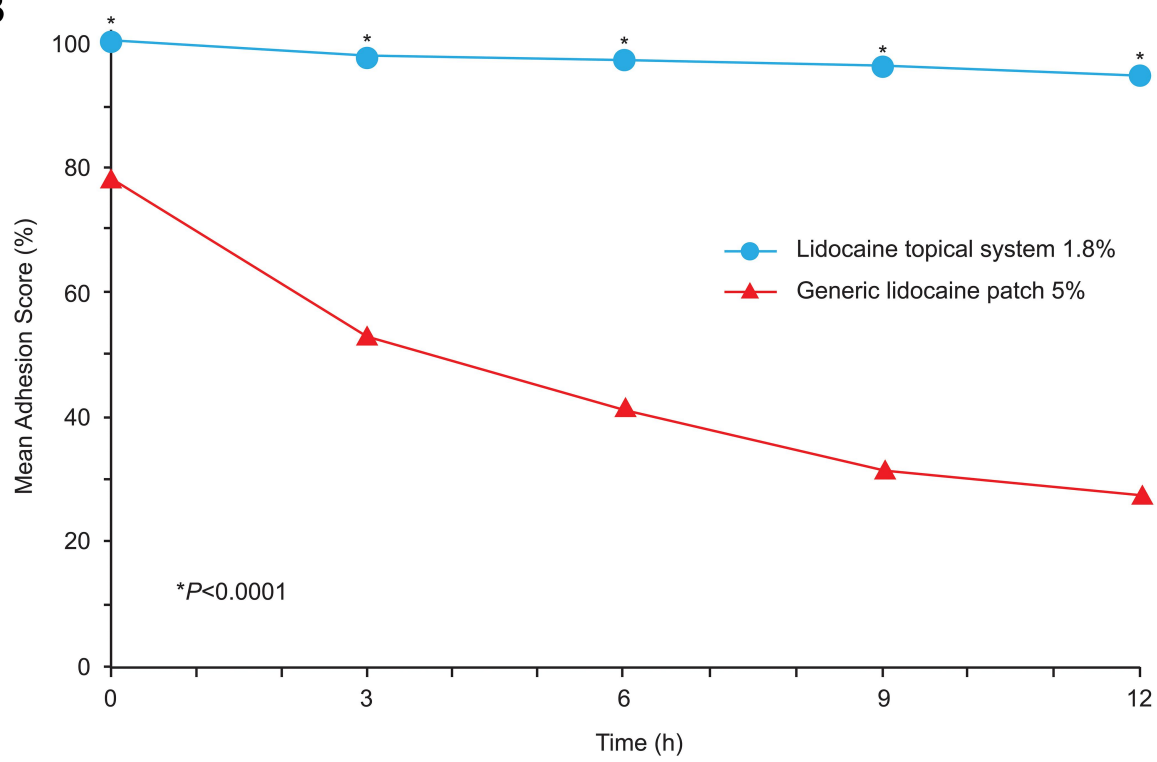

Figure 2 Mean percent adhesion over time. Mean percent adhesion scores were evaluated every 3 hours over the 12-hour application period. (A) In Study 2, there was a significant difference favoring the lidocaine topical system $1.8 \%$ over the lidocaine patch $5 \%$ and lidocaine medicated plaster $5 \%$ at baseline and at each time point after application $(\mathrm{P}<0.000 \mathrm{I})$. (B) In Study 3, there was a significant difference favoring the lidocaine topical system I.8\% over the generic lidocaine patch $5 \%$ at baseline and at each time point after application ( $\mathrm{P}<0.000 \mathrm{I}$ for all comparisons).

0.5 hours, and these cases resolved to scores $\leq 1$ by 2 hours. No subject had a score $>2$ at either time point.

Skin irritation assessments were also performed at 0.5, 2, and 12 hours after product removal in Study 2. All subjects had scores of 0 (no irritation) or 1 at each time point. Mean irritation scores over the entire 12-hour period for all subjects were comparable and not significantly different: $0.114 \pm 0.03186$ for lidocaine topical system $1.8 \%, 0.067 \pm 0.2504$ for lidocaine patch $5 \%$, and $0.065 \pm 0.2478$ for lidocaine medicated plaster $5 \%(P=0.1656)$ (Figure 4).

No AEs were reported during Studies 1 and 2; however, three mild-grade AEs were reported during Study 3.
Two AEs involving pruritus were reported in subjects treated with the lidocaine topical system $1.8 \%$ during the first period of product assessment (one on the left forearm and one inferior to the application site); they resolved completely on follow-up. One AE (mild headache) was reported in a subject treated with the generic lidocaine patch 5\% during the second period of product wear, also with a complete resolution on follow-up. Although formal assessments of skin irritation were not performed, no subjects reported unacceptable skin irritation during use or after removal of the lidocaine topical system $1.8 \%$ or generic lidocaine patch $5 \%$. No serious AEs or deaths 
A

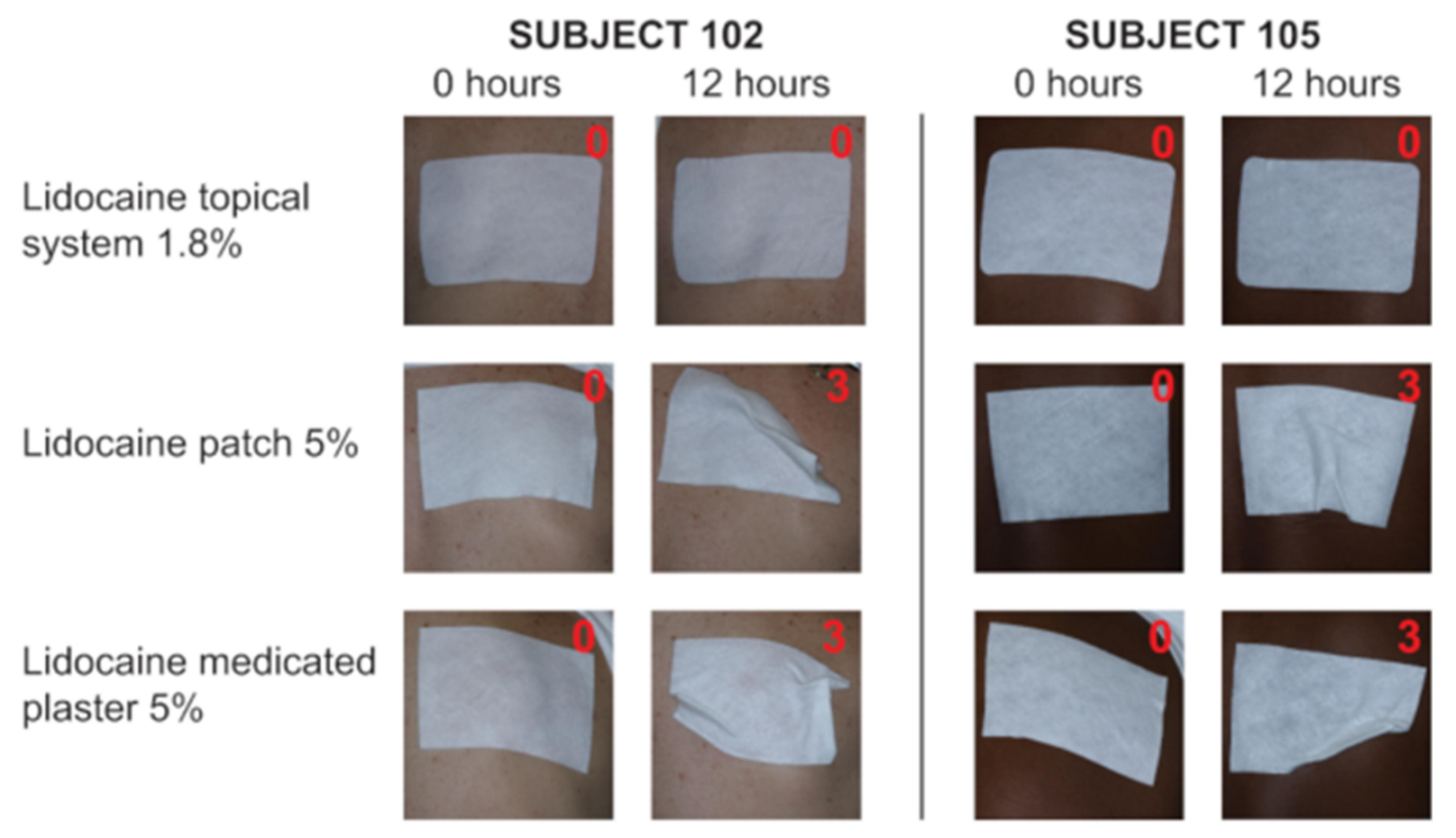

B



Figure 3 Representative adhesion performance with the FDA score (denoted in red) in Studies 2 and 3. Subjects were treated with the lidocaine topical system I.8\% (top rows in panels $\mathbf{A}$ and $\mathbf{B}$ ), lidocaine patch $5 \%$ (middle row in $\mathbf{A}$ ), lidocaine medicated plaster $5 \%$ (bottom row in $\mathbf{A}$ ), or generic lidocaine patch $5 \%$ (bottom row in $\mathbf{B}$ ). Photographs were taken immediately following product application ( 0 hours) and at the end of the study after 12 hours ( \pm 15 minutes) of wear.

were reported throughout the entire study, and no clinically significant findings were reported for any subject who participated in the post-study safety assessments.

\section{Discussion}

Adhesion is a critical attribute of a topical system, in addition to efficacy and safety, and it is important that the product 


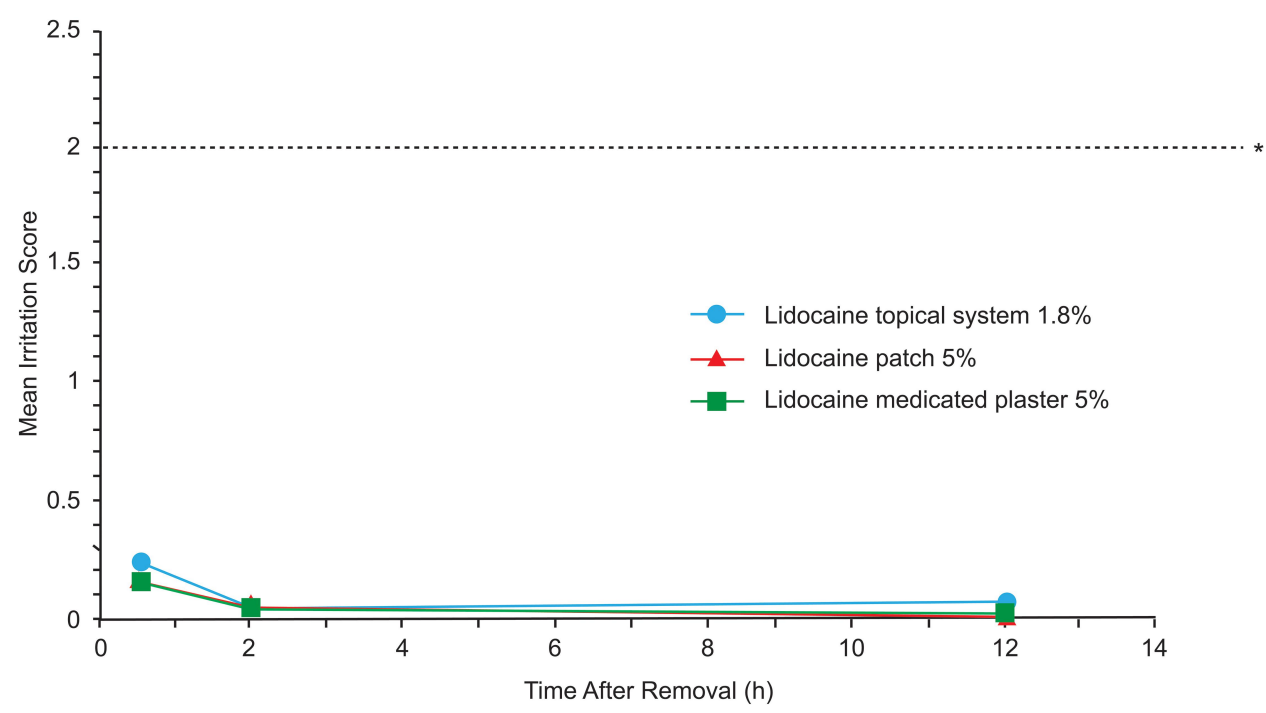

Figure 4 Mean irritation scores after product removal in Study 2. Irritation at the application site was assessed at 0.5 hours ( +10 minutes), 2 hours $( \pm 15$ minutes), and 12 hours ( \pm 30 minutes) after product removal. Irritation was graded using an 8-point scale of dermal response . Overall mean scores across all time points were not significantly different between the lidocaine topical system $1.8 \%$ and lidocaine patch $5 \%$ or lidocaine medicated plaster $5 \%$ ( $P=0.1656$ for both comparisons). *Horizontal line represents a score of 2 , defined as definite erythema, minimal edema, or minimal papular response and is considered clinically meaningful irritation.

maintains adhesion throughout the indicated wear period. The lidocaine topical system 1.8\% met this standard in three different studies and performed better than comparator products with the same indications and wear time (12 hours). This was attributed to its unique drug-in-adhesive nonaqueous polymer system specifically formulated to maintain tackiness over time while delivering comparable levels of the drug to the lidocaine patch $5 \%$ and generic product. To our knowledge, Studies 2 and 3 reported here are the first adhesion performance studies to directly compare four prescription products currently available for the topical delivery of lidocaine. The high degree of adhesion ( $\geq 90 \%)$ observed in more than $90 \%$ of subjects at the end of 12 hours of product administration in Study 1 is a benchmark performance attribute for this product type.

Despite the fact that topical and transdermal drug delivery systems have been in clinical use for several decades, concerns about product adhesion to skin and how this may affect drug dosing persist. ${ }^{20,21}$ The effective dose absorbed from a topical or transdermal system can be affected by many factors, including characteristics related to the product's adhesion performance, the total surface area of application (which may be affected by partial lifting), and the duration of application (affected by total product loss). ${ }^{20}$ Using the parameter of actual percent adhesion, these results demonstrate significant superiority of adhesion performance over 12 hours for the lidocaine topical system $1.8 \%$ versus all 3 comparator products: lidocaine patch $5 \%$, lidocaine medicated plaster $5 \%$, and generic lidocaine patch $5 \%$. Given the improvement in adhesion performance using percent adhesion, the use of percent adhesion assessments in addition to, or in replacement of, the FDA 5-point scale should be considered, especially in comparative studies where the FDA scale may not have sufficient sensitivity to discern meaningful differences in adhesion performance.

Significantly, fewer subjects $(2.3 \%)$ treated with the lidocaine topical system $1.8 \%$ experienced product detachment of $50 \%$ or greater compared with the lidocaine patch $5 \%(20.5 \%)$ or lidocaine medicated plaster 5\% (15.9\%). More than one-third of subjects experienced substantial detachment (reduction to $<10 \%$ adhesion) of the generic lidocaine patch 5\%. No lidocaine topical system $1.8 \%$ became completely detached, whereas 2 detachments (4.5\%) occurred with both the lidocaine patch $5 \%$ and lidocaine medicated plaster 5\%, and 7 detachments (29.1\%) occurred with the generic lidocaine patch $5 \%$.

These differences in adhesion performance are expected to be clinically important. Although up to $30 \%$ of patients might experience poor adhesion and need to replace the comparator prescription lidocaine topical products, our results suggest that less than $1 \%$ of patients would have this problem with the lidocaine topical system 
$1.8 \%$ under the conditions studied. Accordingly, one could anticipate that the number of patients needed to treat to prevent one poor adhesion episode through the utilization of the lidocaine topical system $1.8 \%$ compared with the other products is 25 .

The poor adhesion performance observed for the generic Mylan lidocaine patch 5\% in Study 3 indicates that the study was overpowered relative to the hypothesis that the product would have comparable performance to that established by the Lidoderm Patch 5\% reference product in Study 2; the results were surprising, considering the comparable adhesion data reported in the independent study conducted by Mylan in support of its abbreviated new drug application (ANDA). Although the study designs were comparable, the studies were conducted at separate sites using different subjects using different time points, which could explain the difference. Also, Studies 2 and 3 may differ from the Mylan ANDA study in that subjects were allowed to remain ambulatory during the treatment periods, whereas the FDA adhesion guidance only prevents the use of artificial means to affect adhesion performance (tape reinforcement, overlays, pressing, etc.). ${ }^{24}$ Because patients should expect comparable adhesion performance when switched to generic topical and transdermal products, perhaps this regulatory guidance should be revisited to incorporate design features that more rigorously challenge adhesion performance relative to realworld use.

The lidocaine topical system $1.8 \%$ was well tolerated throughout the studies, with no unacceptable skin irritation necessitating early removal. The mild irritation observed in Studies 1 and 3 shortly after product removal spontaneously resolved within 2 hours, suggesting that this is a transient effect, most likely associated with mechanical removal of the product and improved adhesion. The results confirm the safety and tolerability profile of lidocaine topical patch products. These qualities make lidocaine topical products an attractive option for treating frail and elderly patients with PHN. ${ }^{27}$ The superior adhesion of the lidocaine topical system $1.8 \%$ compared with three comparator lidocaine products may offer patients' advantages with regard to effective drug delivery, safety, and convenience - especially for older individuals with mobility or physical limitations - with the potential to increase patient compliance.

Assessment every 3 hours during the 12-hour periods of product wear throughout these adhesion performance studies is consistent with best practices in FDA guidance and represents a strength of the study design. ${ }^{24}$ The uniform spacing of observations over time is recommended to avoid a disproportionate weighting of adhesion at early time points (when adhesion might be relatively better) and is a key factor for an accurate assessment.

Several limitations of these adhesion performance studies should be mentioned. The volunteer subjects in these studies ranged in age from 18 to 64 (median ages of 47 and 32 years in Studies 2 and 3, respectively) and were younger than the patient population most often diagnosed with PHN-individuals over the age of $60 .^{3}$ These older patients may have distinct skin properties (texture, thickness, moisture, etc.) that could affect adhesion in a realworld setting. ${ }^{28}$ Unlike patients for whom these topical products are indicated, the healthy study subjects did not have or were not recovering from herpes zoster rash. The products studied here were applied to the lower, middle, or upper back, such placement was selected as a standardized area of administration and convenience for frequent assessments. It is not known whether their adhesive capacity can be extrapolated to other anatomical sites.

Finally, throughout the studies, subjects were restricted in performing activities (such as strenuous exercise, exposure to water, and excessive heat or sweating) that could affect product adhesive properties outside a clinical study setting. These restrictions and other factors, such as climate differences (eg, temperature, humidity), may affect the generalizability of the results. In a separate study, the lidocaine topical system $1.8 \%$ was able to withstand conditions of moderate physical exercise (exercise bike) and heat (heating pad) with no meaningful degree of detachment. ${ }^{29}$ In addition, products were only evaluated during 12-hour daylight periods, but many patients with PHN experience nighttime pain and would be expected to use lidocaine products while sleeping. It is not known how these variables would affect the adhesion of the lidocaine topical system $1.8 \%$, nor whether its superior adhesion relative to the other products reported here would be maintained. Regardless, the lidocaine topical system $1.8 \%$ demonstrated superior adhesion to the comparator products tested.

\section{Conclusions}

The lidocaine topical system $1.8 \%$ demonstrated optimum adhesion performance ( $\geq 90 \%$ adhesion) in the majority of subjects during a 12-hour period of administration. Adhesion superiority was consistently demonstrated versus three comparator products-lidocaine patch 5\%, 
lidocaine medicated plaster 5\%, and generic lidocaine patch 5\%-over 12 hours of wear, using actual mean percent adhesion scores and with translation to an adhesion scale. No detachments of the lidocaine topical system $1.8 \%$ occurred. Application and use of all lidocaine topical products were safe and well tolerated. For patients with pain associated with PHN, these results support the use of the lidocaine topical system $1.8 \%$ as an effective and practical treatment that may be preferable to other topical lidocaine options.

\section{Data Sharing Statement}

The authors certify that this manuscript reports original clinical trial data. Individual participant data that underlie the results reported in this article after deidentification (text, tables, and figures) are available, including the study protocol. Data requests should be submitted in the form of a research proposal to medical_affairs@scilexpharma.com for up to 36 months after the publication date.

\section{Acknowledgments}

The authors would like to thank the clinical investigators, clinical staff, and volunteers who made these studies possible. Roger Aitchison of North Rim Consulting provided valuable statistical analyses. Nirzari Parikh, PhD, of Synergy Medical Education provided medical writing support. Dr. Gudin is now affiliated with University of Miami, Miller School of Medicine, Department of Anesthesiology, Perioperative Medicine and Pain Management, Miami, FL, USA.

\section{Funding}

This research was supported by Scilex Pharmaceuticals Inc.

\section{Disclosure}

Dr. Gudin has been an advisor, consultant, speaker bureau member, or shareholder in the past year for Averitas Pharma, BioDelivery Sciences International, Inc., Daiichi Sankyo, Hisamitsu Pharmaceutical Co. Inc., Nektar, Quest Diagnostics, Salix Pharmaceuticals, Scilex Pharmaceuticals Inc., and Virpax Pharmaceuticals. Dr. Webster is affiliated with PRA Health Sciences and has been an advisor or consultant for Alcobra, BioDelivery Sciences International, Inc., Bonti, Charleston Laboratories, Daiichi Sankyo, Depomed, Egalet, Elysium Pharmaceuticals, Ensysce Biosciences, Indivior, Inspirion Pharmaceuticals, Insys Therapeutics, Jefferies, KemPharm, Mallinckrodt Pharmaceuticals, Merck,
Neurana, Pain Therapeutics, Pernix, Pfizer, Salix, Shionogi, Teva, Trevena, Trevi Therapeutics, Vallon, and Vector Pharma. Drs. Greuber, Vought, and Patel are employees of Scilex Pharmaceuticals Inc. Dr. Kuritzky has no relationships to disclose; the opinions in this article do not represent those of his institutional affiliation (University of Florida). The authors report no other conflicts of interest in this work.

\section{References}

1. Centers for Disease Control and Prevention. Shingles (herpes zoster); 2019. Available from: https://www.cdc.gov/shingles/. Accessed March 10, 2020.

2. Hadley GR, Gayle JA, Ripoll J, et al. Post-herpetic neuralgia: a review. Curr Pain Headache Rep. 2016;20(3):17. doi:10.1007/ s11916-016-0548-x

3. Massengill JS, Kittredge JL. Practical considerations in the pharmacological treatment of postherpetic neuralgia for the primary care provider. J Pain Res. 2014;7:125-132. doi:10.2147/JPR. S57242

4. Cohen JI, Solomon CG. Clinical practice: herpes zoster. $N$ Engl J Med. 2013;369(3):255-263. doi:10.1056/NEJMcp1302674

5. Meyers JL, Madhwani S, Rausch D, et al. Analysis of real-world health care costs among immunocompetent patients aged 50 years or older with herpes zoster in the United States. Hum Vaccin Immunother. 2017;13(8):1861-1872. doi:10.1080/21645515.2017.1324373

6. Gudin J, Fudin J, Wang E, et al. Treatment patterns and medication use in patients with postherpetic neuralgia. J Manag Care Spec Pharm. 2019;25(12):1387-1396. doi:10.18553/jmcp.2019.19093

7. Gudin J, Nalamachu S. Utility of lidocaine as a topical analgesic and improvements in patch delivery systems. Postgrad Med. 2020;132 (1):28-36. doi:10.1080/00325481.2019.1702296

8. Davies PS, Galer BS. Review of lidocaine patch $5 \%$ studies in the treatment of postherpetic neuralgia. Drugs. 2004;64(9):937-947. doi:10.2165/00003495-200464090-00002

9. Stanos SP, Galluzzi KE. Topical therapies in the management of chronic pain. Postgrad Med. 2013;125(suppl sup1):25-33. doi:10.1080/00325481.2013.1110567111

10. ZTLIDO ${ }^{\mathrm{TM}}$ (lidocaine topical system) [prescribing information]. San Diego, CA: Scilex Pharmaceuticals Inc; 2018.

11. Fornasari D. Pharmacotherapy for neuropathic pain: a review. Pain Ther. 2017;6(suppl S1):25-33. doi:10.1007/s40122-017-0091-4

12. Leppert W, Malec-Milewska M, Zajaczkowska R, et al. Transdermal and topical drug administration in the treatment of pain. Molecules. 2018;23(3):681. doi:10.3390/molecules23030681

13. LIDODERM $^{\circledR}$ (lidocaine patch 5\%) [prescribing information]. Malvern, PA: Endo Pharmaceuticals Inc.; 2018.

14. US Food and Drug Administration. Drug approval package: lidoderm (lidocaine) patch; 2005. Available from: https://www.accessdata.fda. gov/drugsatfda_docs/nda/99/20612.cfm. Accessed March 10, 2020.

15. VERSATIS ${ }^{\circledR}$ (700 mg medicated plaster). Summary of product characteristics. Uxbridge, UK: Grünenthal Ltd.; 2018

16. Lidocaine patch $5 \%$ [prescribing information]. Parsippany, NJ: Actavis Laboratories UT Inc.; 2015.

17. Lidocaine patch $5 \%$ [prescribing information]. Morgantown, WV: Mylan Pharmaceuticals Inc.; 2018

18. ZTlido - a new lidocaine patch for postherpetic neuralgia. Med Lett Drugs Ther. 2019;61(1568):41-43.

19. Gudin J, Argoff C, Fudin J, et al. A randomized, open-label, bioequivalence study of lidocaine topical system $1.8 \%$ and lidocaine patch 5\% in healthy subjects. J Pain Res. 2020;13:1485-1496. doi:10.2147/JPR.S237934 
20. Wokovich AM, Prodduturi S, Doub WH, et al. Transdermal drug delivery system (TDDS) adhesion as a critical safety, efficacy and quality attribute. Eur $J$ Pharm Biopharm. 2006;64(1):1-8. doi:10.1016/j.ejpb.2006.03.009

21. Cilurzo F, Gennari CG, Minghetti P. Adhesive properties: a critical issue in transdermal patch development. Expert Opin Drug Deliv. 2012;9(1):33-45. doi:10.1517/17425247.2012.637107

22. US Food and Drug Administration. FDA Adverse Events Reporting System (FAERS) public dashboard. Data as of March 31, 2018. Accessed July 27, 2018.

23. World medical association declaration of Helsinki: ethical principles for medical research involving human subjects. JAMA. 2013;310 (20):2191-2194. doi:10.1001/jama.2013.281053

24. US Food and Drug Administration. Guidance for Industry. Assessing adhesion with transdermal and topical delivery systems for ANDAs, October 2018. Accessed June 17, 2019.

25. European Medicines Agency. Guideline on the pharmacokinetic and clinical evaluation of modified release dosage forms, June 2015. Accessed February 19, 2020.
26. US Food and Drug Administration. Guidance for industry. Assessing the irritation and sensitization potential of transdermal and topical delivery systems for ANDAs. Rockville, MD, USA; 2018: Available from: https://www.fda.gov/media/117569/download. Accessed September $16,2020$.

27. Mallick-Searle T, Snodgrass B, Brant JM. Postherpetic neuralgia: epidemiology, pathophysiology, and pain management pharmacology. J Multidiscip Healthc. 2016;9:447-454. doi:10.2147/ JMDH.S106340

28. Wey S-J, Chen D-Y. Common cutaneous disorders in the elderly. $J$ Clin Gerontol Geriatrics. 2010;1(2):36-41. doi:10.1016/j. jegg.2010.10.010

29. Fudin J, Wegrzyn EL, Greuber E, et al. A randomized, crossover, pharmacokinetic and adhesion performance study of a lidocaine topical system $1.8 \%$ during physical activity and heat treatment in healthy subjects. J Pain Res. 2020;13:1359-1367. doi:10.2147/JPR. S238268

\section{Publish your work in this journal}

The Journal of Pain Research is an international, peer reviewed, open access, online journal that welcomes laboratory and clinical findings in the fields of pain research and the prevention and management of pain. Original research, reviews, symposium reports, hypothesis formation and commentaries are all considered for publication. The manuscript management system is completely online and includes a very quick and fair peer-review system, which is all easy to use. Visit http:// www.dovepress.com/testimonials.php to read real quotes from published authors. 